\title{
Atenção pré-natal na rede básica: uma avaliação da estrutura e do processo
}

\author{
Prenatal care at the primary health care level: \\ an assessment of the structure and process
}

Denise Silva da Silveira 1,2

Iná Silva dos Santos 2

Juvenal Soares Dias da Costa 2

1 Secretaria Municipal de Saúde e Bem-Estar de Pelotas. Rua Lobo da Costa 1764, Pelotas, RS 96010-150, Brasil. 2 Departamento de Medicina Social, Faculdade de Medicina, Universidade Federal de Pelotas. Av. Duque de Caxias 250, 2 o andar, Pelotas, $R S$ 96030-002, Brasil.

\begin{abstract}
With the aim of describing the structure and process by which prenatal care is provided at the primary health care level in the city of Pelotas in southern Brazil, a descriptive crosssectional study was conduced. All 31 urban health care facilities were visited and their structure analyzed. A total of 839 prenatal records from mothers who delivered in the six months prior to the beginning of the study were reviewed. The structure as a whole was classified as poor (only 70\% of standard parameters were present), mainly due to deficiencies in the physical plant. Process assessment showed low coverage (53\%) and an average of 5.3 visits. Only $37 \%$ of patient records showed adequate results (Kessner Index). Adding laboratory test results and routine procedures as complementary criteria, 31\% and 5\% of the records scored as adequate, respectively. In summary, overall quality of prenatal care provided at the primary health care level in Pelotas is poor. Mechanisms to increase staff adherence to both procedures and program logistics need to be developed.
\end{abstract}

Key words Prenatal Care; Hospitalization; Hospitalized Child

Resumo Realizou-se estudo transversal descritivo e auditoria de registros médicos para avaliar a estrutura e o processo de atendimento pré-natal nas unidades de atenção primária à saúde em Pelotas, Sul do Brasil. Para a investigação da estrutura, foram incluídas todas as 31 unidades da zona urbana. No estudo do processo revisou-se os registros de pré-natal das mães com data provável de parto nos 6 meses anteriores ao início do trabalho de campo, num total de 839 formulários. A estrutura foi considerada precária (70\% da ideal), principalmente devido a deficiências da planta física. Quanto ao processo, observaram-se uma baixa cobertura (53\%) e média de consultas de 5,3. O pré-natal foi adequado em somente 37\% dos registros (Índice de Kessner). Adicionando-se exames laboratoriais e procedimentos da consulta como critérios complementares, $31 \%$ e 5\% dos registros foram considerados adequados, respectivamente. De um modo geral, a qualidade do cuidado pré-natal oferecido foi precária. Mecanismos que aumentem a aderência das equipes de saúde aos procedimentos e à lógica do programa, necessitam ser desenvolvidos.

Palavras-chave Cuidado Pré-Natal; Hospitalização; Criança Hospitalizada 


\section{Introdução}

A aplicação dos conceitos e métodos epidemiológicos na avaliação de serviços, programas e tecnologias vem notadamente se desenvolvendo, especialmente com relação a investigações sobre a qualidade da atenção médica (Fosberg et al., 1991; Black, 1994; Goldbaum, 1996).

Para estimar a qualidade dos serviços, Donabedian (1988) tem recomendado a análise da estrutura, do processo e do resultado, considerando o estudo do processo como a melhor forma para investigar-se o conteúdo da atenção oferecida no primeiro nível de assistência.

Com relação à qualidade da atenção dedicada ao pré-natal, os principais problemas apontados em estudos da literatura referem-se ao não-cumprimento das normas e rotinas por parte dos profissionais, ao não-preenchimento de registros e à constatação de que os cuidados dispensados são inversamente direcionados às necessidades (Hansell, 1991; Peoples-Sheps et al., 1991; Petitti et al., 1991; Camús et al., 1992; Kogan et al., 1994; Sikosana, 1994).

Em nosso país, estudos que visem avaliar a qualidade dos serviços são escassos. Na sua maioria, limitam-se a descrever características da atenção médica, tais como cobertura, número de consultas pré-natais e tipo de parto (Tanaka et al., 1989; Gomes et al., 1990; Scochi, 1991). Apenas um estudo (Takeda, 1993) investigou a qualidade desse cuidado, empregando alguns indicadores de adequação, entre eles o Índice de Kessner, modificado pela autora, e a verificação do conteúdo das consultas.

Os resultados do estudo da coorte de Pelotas de 1993 (Halpern et al., 1998) mostraram que, apesar da alta cobertura pré-natal no município, existem desigualdades nos cuidados oferecidos às gestantes. As de maior risco gestacional apresentaram maior proporção de prénatal classificado como inadequado ou intermediário (25\%), quando comparadas com as de menor risco (10\%). Aquelas que não receberam nenhum atendimento antes do parto eram mais pobres, adolescentes ou com idade acima de quarenta anos e tiveram uma incidência de baixo peso ao nascer 2,5 vezes maior do que as do grupo de mães que realizaram cinco ou mais consultas.

No momento, o Município de Pelotas caminha para o processo de municipalização plena da atenção básica à saúde (SMSBE, 1996), sendo responsável pelo gerenciamento de 31 unidades de saúde, localizadas na zona urbana, e 12 na zona rural. Nesses serviços, desenvolvemse ações através de programas com ênfase no grupo materno-infantil, onde o atendimento ao pré-natal é uma ação prioritária.

Esse estudo teve como objetivo descrever a atenção pré-natal oferecida às gestantes usuárias desses serviços, utilizando para tal a caracterização da estrutura e do processo de atendimento. Espera-se que as conclusões, cientificamente embasadas, possam ser utilizadas pelos gestores de saúde locais, a fim de alcançar ações mais efetivas e de melhor qualidade.

\section{Material e métodos}

O estudo foi composto por dois subestudos complementares: um estudo descritivo, para avaliação da estrutura das unidades, e uma auditoria de registros médicos do atendimento pré-natal, para avaliação do processo.

\section{Estrutura}

O subestudo da estrutura incluiu todas as 31 unidades de saúde da Secretaria Municipal de Saúde e Bem-Estar (SMSBE) de Pelotas localizadas na zona urbana do município. Foi conduzido durante o mês de maio de 1998, com aplicação, em cada unidade, do Instrumento de Avaliação para Centros e Postos de Saúde (MS, 1985). Nesse instrumento, centros de saúde são definidos como unidades operadas por médicos, e postos de saúde, como unidades sem médicos, operadas por pessoal de nível elementar. Uma vez que todas as unidades de saúde eram operadas por médicos, aplicou-se o instrumento para avaliação de centros de saúde, que diferenciou os serviços, quanto ao porte, em três tipos: tipo 1 (CS1) - com um consultório médico; tipo 2 (CS2) - com dois consultórios médicos e tipo 3 (CS3) - com três consultórios médicos.

Áreas-alvo da avaliação: planta física, recursos humanos, recursos materiais, normas, atividades e procedimentos e sistema de referência.

Cada área recebeu uma pontuação:

- Planta física (máximo 150 pontos) = atenção à clientela, apoio à planta física, acabamento $\mathrm{e}$ instalações sanitárias.

- Recursos humanos (máximo 300 pontos) = lotação, capacitação e política de pessoal.

- Recursos materiais (máximo 150 pontos) = consultório de ginecologia e obstetrícia, consultório de pediatria, outros materiais necessários. - Normas, atividades e procedimentos (máximo 350 pontos) = normas gerais, de assistência à mulher, de assistência à criança, de controle de doenças transmissíveis e demais atividades. 
- Sistema de referência (máximo 50 pontos). Mil era o máximo de pontos que um serviço poderia alcançar. De acordo com a soma total de pontos obtidos, as unidades foram classificadas em ótimas (900 a 1.000 pontos ou $90 \%$ a $100 \%$ ); satisfatórias (750 a 899 pontos ou $75 \%$ a $89,9 \%$ ); precárias (500 a 749 pontos ou $50 \%$ a $74,9 \%$ ) ou insuficientes (499 e menos pontos ou $49,9 \%$ e menos).

As informações para a avaliação da estrutura foram obtidas por entrevista com os chefes dos postos e por medições da área física, utilizando-se trenas, de acordo com as normas especificadas no instrumento.

\section{Processo}

A auditoria foi realizada mediante a análise da ficha de pré-natal ou, na falta dessa, do prontuário médico, com a aplicação de um instrumento padronizado (ficha de auditoria médica), especialmente elaborado para a extração das informações. Incluíram-se apenas as 26 unidades com atendimento pré-natal.

O trabalho de campo transcorreu de junho a novembro de 1998, analisando-se os registros de pré-natal das mulheres com data provável de parto (DPP) nos seis meses anteriores ao início da coleta de dados, no serviço estudado.

Para calcular a cobertura, relacionou-se o número de registros encontrados com o número de gestantes esperado, em torno de $2 \%$ da população de abrangência dessas 26 unidades de saúde.

Foram coletadas as seguintes informações: número de consultas realizadas durante o prénatal, considerando-se consulta somente quando estivessem registrados pelo menos dois procedimentos; idade gestacional na primeira consulta (quando ausente, era calculada a partir da data da última menstruação ou estimada por meio das informações de idade gestacional nas consultas subseqüentes), expressa em semanas; número de fatores de risco registrados (idade menor de 16 anos ou maior de trinta; problemas mentais; hipertensão; diabetes; aborto ou óbito fetal anterior; hábito de fumar até dez cigarros por dia ou mais de dez por dia; álcool; renda familiar; drogas e doenças venéreas) como presentes ou ausentes. Verificou-se ainda o número de vezes em que os procedimentos do exame clínico e obstétrico (idade gestacional, peso, pressão arterial, altura uterina, ausculta dos batimentos cardiofetais, edema e apresentação), assim como os exames laboratoriais de rotina (hemoglobina, sorologia para sífilis e exame comum de urina) foram registrados, separadamente. Finalmente, os re- gistros do fator Rh, da revisão puerperal, da data de realização do último exame citopatológico, da vacinação antitetânica (VAT) - número de doses da vacina feitas antes do atual pré-natal, tempo decorrido desde a última dose e doses aplicadas durante o pré-natal - e de medicamentos prescritos também foram coletados.

Algumas variáveis foram dicotomizadas: vacina em dia e atrasada, assumindo-se que os valores "não lembra" e "ignorado" para o número de doses prévias corresponderiam a nenhuma dose; e revisão puerperal realizada ou não, assumindo-se que ausência de registro corresponderia à não realização.

A adequação do acompanhamento pré-natal foi avaliada de três formas:

- Combinando-se o número de consultas com a época do início do pré-natal de acordo com o Índice de Kessner modificado por Takeda (1993): adequado = seis ou mais consultas e início pré-natal antes de vinte semanas; inadequado = início do pré-natal após 28 semanas, ou menos de três consultas; intermediário = demais situações.

- Acrescentando-se ao Índice de Kessner modificado o número de vezes em que os exames complementares: hemoglobina (Hb), sorologia para sífilis (VDRL) e exame comum de urina (ECU) foram registrados juntos, ficando a adequação assim definida: adequado = seis ou mais consultas, início pré-natal antes de vinte semanas e um mínimo de dois registros de cada um dos três exames; inadequado = início do pré-natal após 28 semanas, ou menos de três consultas, ou nenhum exame registrado; intermediário = demais situações.

- Acrescentando-se ao anterior o número de vezes em que os procedimentos da consulta pré-natal (altura uterina, apresentação fetal, batimentos cardiofetais, edema, idade gestacional, pressão arterial e peso) foram registrados.

Para compor este último critério, foram estabelecidos pontos de corte. Considerou-se adequado quando foram verificados cinco ou mais registros da altura uterina, idade gestacional, pressão arterial, edema e peso; quatro ou mais registros dos batimentos cardiofetais e dois ou mais registros da apresentação fetal. Considerou-se inadequado quando foram verificados dois ou menos registros da altura uterina, idade gestacional, pressão arterial, batimentos cardiofetais, edema e peso, sem qualquer registro da apresentação fetal.

Os pontos de corte foram definidos da seguinte maneira: uma vez que altura uterina, edema, idade gestacional, pressão arterial e peso independem da duração da gestação para serem verificados e que o número mínimo ade- 
quado de consultas eram seis, aceitou-se como adequado se esses procedimentos tivessem sido registrados pelo menos cinco vezes. Quanto aos batimentos cardiofetais, tendo em vista que, a partir de vinte semanas, é possível auscultá-los com estetoscópio de Pinard (SSMA, 1997) e que, de acordo com as normas do programa de pré-natal vigente, deveriam ser realizadas pelo menos quatro consultas a partir deste período, considerou-se adequado quando se verificaram quatro ou mais registros. Quanto à apresentação fetal, sabendo-se que, a partir de 28 semanas, já é possível avaliá-la clinicamente e que, de acordo com as normas do programa, deveriam ser realizadas pelo menos duas consultas a partir desse período, dois ou mais registros foram considerados adequados.

Os dados foram digitados em dupla entrada, limpos e editados através do programa EPIINFO 6.01 (Dean, 1994).

\section{Resultados}

\section{Estrutura}

As Tabelas 1 e 2 apresentam os resultados da avaliação da estrutura.

Os serviços, como um todo, apresentaram uma média global de pontos de 698 (70\%), sendo classificados como precários. Considerando-se somente as unidades que realizavam prénatal, a média passou para 729 (73\%), sendo igualmente classificadas como precárias (Tabela 1$)$.

Observou-se, também, que a média total de pontos, incluindo todos os serviços, variou de acordo com o porte dos centros de saúde, o que classificou as unidades tipo 1 e 2 como precárias, e as unidades tipo 3, como satisfatórias.

Para todos os serviços, independente do porte, a área com o pior resultado foi a planta física, atingindo somente $38 \%$ do padrão estabelecido pelo instrumento de avaliação.

Na Tabela 2, observa-se que, entre as 31 unidades de saúde estudadas, encontraram-se, quanto ao porte, cinco do tipo CS1, 19 do tipo CS2 e sete do tipo CS3. Das unidades com prénatal, a maioria era de porte médio (CS2), nenhuma tendo sido classificada como insuficiente e mais da metade (14) sendo avaliadas como precárias.

Especificamente, do ponto de vista do cuidado pré-natal, embora a classificação da planta física tenha sido insuficiente, o tamanho dos consultórios médicos resultou em satisfatório. Entre os recursos humanos, o número de médicos foi avaliado, também, como satisfatório, apesar de o número de dentistas ter sido considerado precário.

Quanto aos recursos materiais, os serviços que faziam pré-natal possuíam recursos satisfatórios e ótimos para o atendimento à gestante, exceto quanto a medicamentos, pontuados como insuficientes (analgésicos) e precários (antibióticos e sulfato ferroso).

As principais deficiências na área de normas, atividades e procedimentos, pontuada como satisfatória, estiveram relacionadas com a inexistência de: programação anual das atividades a serem desenvolvidas pelo serviço; avaliação interna; supervisão central e interna; manutenção do sistema de informação por meio dos boletins de notificação de doenças; manutenção de cadastro domiciliar atualizado; triagem da demanda; visitas domiciliares; atividades de grupos e de educação em saúde. Para normas de atenção às gestantes, somam-se as dificuldades quanto à utilização da curva ponderal e à assistência odontológica.

Finalmente, com relação ao sistema de referência, também considerado satisfatório, foram apontadas como deficientes a contra-referência das pacientes e a disponibilidade de leitos para internação de todas as gestantes. O apoio diagnóstico, no que se refere a exames laboratoriais, foi considerado ótimo.

\section{Processo}

Um total de 839 registros de atendimento prénatal foram localizados durante o estudo. Esse número representa uma cobertura de $53 \%$ das gestantes esperadas, considerando-se os seis meses da investigação.

Com relação à fonte da informação, observaram-se $743(88,6 \%)$ registros consultando-se a ficha de pré-natal adotada pelo programa de pré-natal da SMSBE e $96(11,4 \%)$, através dos prontuários médicos.

A média de consultas por gestante foi de 5,3, e a da idade gestacional, no início do prénatal, foi de 17,8 semanas. Aproximadamente $46 \%$ das gestantes ingressaram no programa durante o primeiro trimestre, $41 \%$ no segundo e $13 \%$ no terceiro, com ausência da informação sobre a idade gestacional em $5 \%$ dos registros. Verificou-se, ainda, que $63 \%$ das gestantes iniciaram o pré-natal antes de vinte semanas de gestação.

Ao se combinar a idade gestacional de ingresso no programa com o número de consultas, foi possível classificar o pré-natal de 797 gestantes.

De acordo com o Índice de Kessner modificado por Takeda, encontraram-se $37 \%$ de pré- 
Distribuição dos pontos alcançados por área por todas as unidades, pelas unidades que realizavam pré-natal e por tipo de centro de saúde. Pelotas, Rio Grande do Sul, 1998.

\begin{tabular}{|c|c|c|c|c|c|c|c|c|c|c|c|}
\hline \multirow[t]{2}{*}{ Área } & \multirow{2}{*}{$\begin{array}{l}\text { Pontuação } \\
\text { máxima }\end{array}$} & \multicolumn{2}{|c|}{ Todas } & \multicolumn{2}{|c|}{ Com PN1 } & \multicolumn{2}{|c|}{$\mathrm{CS} 12$} & \multicolumn{2}{|c|}{$\mathrm{CS}^{3}$} & \multicolumn{2}{|c|}{$\mathrm{CS}^{4} 4$} \\
\hline & & $\mathrm{n}$ & $\%$ & $\mathrm{n}$ & $\%$ & $\mathrm{n}$ & $\%$ & $\mathrm{n}$ & $\%$ & $\mathrm{n}$ & $\%$ \\
\hline Planta física & 150 & 57 & 38 & 58 & 39 & 54 & 36 & 53 & 35 & 68 & 45 \\
\hline Recursos humanos & 300 & 220 & 73 & 225 & 73 & 217 & 72 & 215 & 67 & 234 & 78 \\
\hline Recursos materiais & 150 & 109 & 73 & 113 & 75 & 109 & 73 & 107 & 71 & 115 & 76 \\
\hline Normas & 350 & 276 & 78 & 295 & 84 & 235 & 67 & 278 & 79 & 300 & 86 \\
\hline Sistema de referência & 50 & 36 & 72 & 38 & 77 & 30 & 61 & 37 & 74 & 38 & 77 \\
\hline Total & 1.000 & 698 & 70 & 729 & 73 & 645 & 65 & 690 & 69 & 755 & 76 \\
\hline
\end{tabular}

1 PN: Pré-natal.

2 CS1: Centro de saúde de porte 1.

3 CS2: Centro de saúde de porte 2.

4 CS3: Centro de saúde de porte 3.

Tabela 2

Classificação dos tipos de centro de saúde quanto à pontuação para todas as unidades, para as que realizavam pré-natal e por tipo de centro de saúde. Pelotas, Rio Grande do Sul, 1998.

\begin{tabular}{|c|c|c|c|c|c|c|c|c|c|c|}
\hline \multirow[t]{3}{*}{ Tipo de CS } & \multicolumn{2}{|c|}{ № de unidades } & \multicolumn{4}{|c|}{ Precário } & \multicolumn{4}{|c|}{ Satisfatório } \\
\hline & \multirow[t]{2}{*}{ Todas } & \multirow[t]{2}{*}{ Com PN1 } & \multicolumn{2}{|c|}{ Todas } & \multicolumn{2}{|c|}{ Com PN } & \multicolumn{2}{|c|}{ Todas } & \multicolumn{2}{|c|}{ Com PN } \\
\hline & & & $\mathrm{n}$ & $\%$ & $\mathrm{n}$ & $\%$ & $\mathrm{n}$ & $\%$ & $n$ & $\%$ \\
\hline $\mathrm{CS} 12$ & 5 & 3 & 3 & 60 & 1 & 33 & 2 & 40 & 2 & 67 \\
\hline $\mathrm{CS}_{23}$ & 19 & 16 & 13 & 68 & 10 & 63 & 6 & 32 & 6 & 37 \\
\hline CS34 & 7 & 7 & 3 & 43 & 3 & 43 & 4 & 57 & 4 & 57 \\
\hline Total & 31 & 26 & 19 & 61 & 14 & 54 & 12 & 39 & 12 & 46 \\
\hline
\end{tabular}

1 PN: Pré-natal.

2 CS1: Centro de saúde de porte 1.

3 CS2: Centro de saúde de porte 2.

4 CS3: Centro de saúde de porte 3.

natais adequados, 38\% intermediários e $25 \%$ inadequados. Ao adicionarmos exames laboratoriais, verificaram-se $31 \%$ de pré-natais adequados, $42 \%$ intermediários e $27 \%$ inadequados. Acrescentando-se procedimentos da consulta aos critérios anteriores, $5 \%$ dos pré-natais foram classificados como adequados, $91 \%$ como inadequados e $4 \%$ como intermediários.

A Tabela 3 descreve os procedimentos da consulta pré-natal, quanto ao número de vezes em que foram registrados.

Para os procedimentos não dependentes de algum período específico da gravidez para a sua realização, tais como idade gestacional, pressão arterial e peso, a freqüência de registro esperada (cinco ou mais) foi verificada em menos de $50 \%$ dos prontuários. Apenas $16 \%$ das fichas pré-natais continham cinco ou mais registros de pesquisa de edema. O peso da gestante, a pressão arterial e a altura uterina foram as informações com menor percentual de ausência de registro.

A Tabela 4 mostra a freqüência com que foram registrados os exames laboratoriais básicos. Constatou-se que não houve registro da realização de $\mathrm{Hb}$, ECU e VDRL em 14,4\% das gestantes. Semelhante ao verificado com os procedimentos, menos da metade das gestantes $(48,1 \%)$ teve dois ou mais registros dos três exames. O resultado do Fator Rh era conhecido em $86,3 \%$ dos casos. Informação sobre o exame citopatológico foi registrada em $53,4 \%$ dos atendimentos, $35,9 \%$ das gestantes estando em dia (Day, 1986).

Com relação a fatores de risco, em 313 formulários $(37,3 \%)$ não foi possível avaliar a informação por ausência de registro. Dos 526 casos em que houve registro do fator de risco, a 
Tabela 3

\begin{tabular}{|c|c|c|}
\hline Procedimentos de exame clínico & $\mathbf{n}$ & $\%$ \\
\hline \multicolumn{3}{|l|}{ Altura uterina } \\
\hline Nenhuma vez registrada & 60 & 7,2 \\
\hline Pelo menos uma vez registrada & 779 & 92,8 \\
\hline Cinco ou mais vezes registrada & 413 & 49,2 \\
\hline \multicolumn{3}{|l|}{ Apresentação } \\
\hline Nenhuma vez registrada & 421 & 50,2 \\
\hline Pelo menos uma vez registrada & 418 & 49,8 \\
\hline Duas ou mais vezes registrada & 282 & 33,6 \\
\hline \multicolumn{3}{|l|}{ Batimento cardiofetal } \\
\hline Nenhuma vez registrado & 139 & 16,6 \\
\hline Pelo menos uma vez registrado & 700 & 83,4 \\
\hline Quatro ou mais vezes registrado & 427 & 50,9 \\
\hline \multicolumn{3}{|l|}{ Edema } \\
\hline Nenhuma vez registrado & 506 & 60,3 \\
\hline Pelo menos uma vez registrado & 333 & 39,7 \\
\hline Cinco ou mais vezes registrado & 133 & 15,9 \\
\hline \multicolumn{3}{|l|}{ Idade gestacional } \\
\hline Nenhuma vez registrada & 112 & 13,3 \\
\hline Pelo menos uma vez registrada & 727 & 86,7 \\
\hline Cinco ou mais vezes registrada & 376 & 44,8 \\
\hline \multicolumn{3}{|l|}{ Pressão arterial } \\
\hline Nenhuma vez registrada & 44 & 5,2 \\
\hline Pelo menos uma vez registrada & 795 & 94,8 \\
\hline Cinco ou mais vezes registrada & 413 & 49,2 \\
\hline \multicolumn{3}{|l|}{ Peso } \\
\hline Nenhuma vez registrado & 42 & 5,0 \\
\hline Pelo menos uma vez registrado & 797 & 95,0 \\
\hline Cinco ou mais vezes registrado & 406 & 48,4 \\
\hline
\end{tabular}

freqüência de nenhum fator identificado foi de $19,0 \%$, e a de tabagismo materno foi de $36,1 \%$.

Quanto ao número de doses prévias da VAT, o percentual de ausência de informação foi de $41 \%$, e o de tempo decorrido desde a última dose não foi registrado em $52 \%$. Considerando-se o número de doses prévias ignorado como zero dose e não se levando em conta a informação sobre tempo decorrido desde a última dose, $55 \%$ das gestantes foram adequadamente imunizadas no pré-natal.

Os medicamentos mais prescritos, entre os registrados (406), foram sulfato ferroso (62\%), hioscina (20\%) e ampicilina (18\%). A revisão puerperal foi registrada como realizada em apenas $32,5 \%$ das fichas, sendo o restante da informação ignorada.

\section{Discussão}

Entre as limitações, destacam-se a aplicação de um instrumento não específico para pré-natal na avaliação da estrutura e a utilização de dados secundários para avaliação do processo. $\mathrm{O}$ uso de dados secundários não permite estimar o quanto a freqüência de sub-registro distorce os resultados encontrados.

A utilização do instrumento para avaliação da estrutura, proposto pelo Ministério da Saúde, permitiu conhecer com detalhes a situação dos serviços estudados.

Entre as unidades que prestavam cuidado pré-natal, a maioria foi classificada como precária, sendo a planta física a área mais deficitária, sugerindo que foram idealizadas sem um planejamento de construção adequado aos padrões estabelecidos pelo Ministério da Saúde. Considerando-se um mínimo de estrutura física para o atendimento pré-natal, o tamanho dos consultórios foi satisfatório, não havendo impedimentos à sua realização. No entanto, a área física de suporte mostrou-se bastante deficiente.

A área de recursos humanos, na atenção à gestante, alcançou um escore satisfatório para o número de médicos e precário quanto ao número de dentistas, com possíveis limitações à cobertura do programa e à assistência odontológica de todas as gestantes.

Ao se considerarem as demais áreas satisfatórias (recursos materiais; normas, atividades e procedimentos; sistema de referência) quanto ao cuidado pré-natal, os principais problemas encontrados foram: falta de medicamentos, impossibilitando o tratamento adequado de intercorrências comuns da gravidez; carência de uma visão coletiva no planejamento das ações de saúde do serviço, o que pode interferir na execução das normatizações; insuficiência da contra-referência, limitando o acompanhamento dessas pacientes pela unidade de saúde e favorecendo o abandono; insuficiência de leitos para gestantes, facilitando desfechos desfavoráveis.

A Organização Pan-Americana da Saúde (OPAS), em 1985, desenvolveu uma metodologia para avaliação de serviços de saúde que aborda o tema de forma análoga ao instrumento utilizado neste estudo. Resultados de sua aplicação em 18 países da América Latina (Oje- 
da, 1992) mostraram semelhantes deficiências na estrutura dos serviços de atenção maternoinfantil, mesmo adotando pontos de corte menos exigentes na definição das categorias.

Espera-se que serviços com uma melhor estrutura apresentem um melhor processo de atendimento. Porém, essa condição não é essencial, uma vez que a relação entre esses dois elementos depende também das inter-relações pessoais entre profissionais e gestores do sistema, componente que não foi avaliado por este estudo.

A cobertura de gestantes encontrada pela rede de serviços (53\%) é consistente com o resultado de um recente estudo local (dados ainda não publicados) e superior às relatadas em outros estudos brasileiros (Tanaka et al., 1989; Scochi, 1994). Como a cobertura pré-natal no Município de Pelotas é bastante alta (95\%), segundo dados da coorte de 1993 (Halpern et al., 1998), e, já que não existem mecanismos de regionalização nem hierarquização do atendimento, é provável que exista evasão de gestantes para outros serviços. É possível também que esteja ocorrendo sub-registro da inscrição de gestantes no programa de pré-natal das unidades de saúde da SMSBE.

Apesar de estar normatizada a utilização da ficha de pré-natal para registro das informações das consultas, três serviços não a adotavam. Entende-se que a padronização facilita e uniformiza as anotações, assim como favorece a realização de auditorias.

O presente estudo encontrou média de 5,3 consultas durante o pré-natal, resultado inferior ao do estudo da coorte de 1993 de Pelotas (Halpern et al., 1998), cujo valor era de 7,6. Possivelmente, a diferença em relação à coorte se deva à ocorrência de três fatores fundamentais: as gestantes usuárias do pré-natal das unidades da SMSBE são as de mais baixa renda, caracterizando-se como as que consultam menos (Costa et al., 1996); sub-registro das consultas realizadas, principalmente a primeira, quando são solicitados os exames; dificuldade de acesso em virtude de os serviços funcionarem, na sua maioria, somente nos turnos diurnos. Em média, no entanto, o programa está atingindo o número mínimo de consultas preconizado pelo Programa de Pré-natal de Baixo-Risco do Ministério da Saúde. Essa média foi mais consistente com a encontrada em outros estudos (Takeda et al., 1993; Costa et al., 1996) que investigaram populações semelhantes.

De acordo com o critério de adequação utilizado, encontrou-se uma proporção de prénatal adequado de $37 \%$, inferior à encontrada no estudo da coorte de 1993 (Halpern et al.,

\begin{tabular}{|c|c|c|}
\hline \multicolumn{3}{|c|}{$\begin{array}{l}\text { Características dos exames laboratoriais da consulta } \\
\text { pré-natal. Pelotas, Rio Grande do Sul, } 1998 .\end{array}$} \\
\hline Exames laboratoriais & $\mathbf{n}$ & $\%$ \\
\hline \multicolumn{3}{|c|}{ № de Hb/ECU/VDRL no pré-natal1 } \\
\hline Nenhuma vez registrados & 121 & 14,4 \\
\hline $1 \mathrm{vez}$ registrados & 314 & 37,4 \\
\hline 2 vezes registrados & 289 & 34,4 \\
\hline 3 ou mais vezes registrados & 115 & 13,7 \\
\hline \multicolumn{3}{|l|}{ Fator $\mathrm{Rh}$} \\
\hline Com registro & 724 & 86,3 \\
\hline Sem informação & 115 & 13,7 \\
\hline \multicolumn{3}{|l|}{ Citopatológico } \\
\hline Em dia & 301 & 35,9 \\
\hline Atrasado & 18 & 2,1 \\
\hline Nunca fez & 111 & 13,2 \\
\hline Não lembra & 18 & 2,1 \\
\hline Sem informação & 391 & 46,6 \\
\hline
\end{tabular}

$1 \mathrm{Hb}=$ hemoglobina; $\mathrm{ECU}=$ exame comum de urina; $\mathrm{VDRL}=$ sorologia para sífilis .

1998), de $82,7 \%$, sendo aquela mais consistente com a de estudos que analisaram populações do estrato mais pobre (Takeda et al., 1993; Costa et al., 1996), confirmando, portanto, a conhecida lei dos cuidados inversos. Destacouse, ainda, a elevada proporção de pré-natal classificado como inadequado.

Esses achados podem estar refletindo problemas tanto do serviço, quanto do usuário. Quanto ao serviço, as equipes podem não estar preparadas para implementar ações de divulgação do programa, para trabalhar com prioridades, para buscar gestantes faltosas e para interagir como equipe multidisciplinar. Quanto às usuárias, pode estar ocorrendo uma falta de conscientização sobre a importância do ingresso precoce no pré-natal e do comparecimento às consultas, principalmente entre as gestantes de baixa renda.

O número de consultas pré-natais não garante a qualidade do cuidado. Quando acrescentou-se ao critério de adequação (Índice de Kessner modificado por Takeda) um número mínimo de registro de exames, observou-se que a proporção de pré-natal adequado ficou ainda menor, permitindo uma visão mais qualitativa da atenção oferecida. Quando adicionaram-se procedimentos ao critério anterior (Kessner modificado e exames complementares), o índice de adequação foi excepcionalmente baixo (5\%), com uma inadequação de $91 \%$. 
Examinando-se as características dos procedimentos considerados como importantes no pré-natal, encontramos uma baixa freqüência de registro quando comparada com a de outros estudos (Hansell, 1991; Peoples-Sheps, 1991; Camús et al., 1992; Takeda et al., 1993; Sikosana, 1994), em parte porque podem não estar sendo realizados em todos os momentos e, em parte, em razão do sub-registro das informações.

Quanto aos exames de rotina, encontrou-se elevado percentual de ausência de registro, superior ao encontrado por outros autores (Hansell, 1991; Peoples-Sheps, 1991; Camús et al., 1992; Sikosana, 1994), necessitando-se avaliar se realmente não foram solicitados, se foram solicitados e não realizados, se ocorreu sub-registro da informação ou se não houve tempo de realizar exames por ingresso tardio no prénatal. A baixa freqüência encontrada para o registro dos três exames, em três oportunidades diferentes, pode ser explicada pelos mesmos fatores, acrescentando-se, ainda, a possibilidade da ocorrência de interrupção precoce da gravidez ou de parto prematuro.

É expressiva a proporção de sub-registro para apresentação fetal, edema, ano de realização do último exame citopatológico, revisão puerperal e vacinação antitetânica. Mesmo assim, os resultados do exame Papanicolau em dia, da revisão puerperal realizada e da imunização para a VAT foram consistentes com estudos locais (Costa et al., 1996; Halpern et al., 1998).

\section{Conclusões}

Esforços devem ser feitos para melhorar a qualidade da atenção oferecida por esses serviços. É necessário, portanto, ampliar a cobertura do programa, motivar e capacitar os profissionais para a prática da saúde coletiva e trabalho em equipe multidisciplinar, garantir a realização dos procedimentos da consulta de pré-natal, garantir o tratamento das intercorrências comuns da gravidez e organizar o sistema de atendimento entre os níveis.

Enquanto não se dispõe de avaliações que investiguem os fatores associados não só à baixa adesão dos profissionais aos registros e, provavelmente, à realização dos procedimentos recomendados da consulta, como também à baixa adesão das mães ao programa de pré-natal da rede pública, os resultados de estudos locais de monitorização do programa devem ser amplamente discutidos com as equipes de saúde a fim de otimizar os recursos materiais e humanos disponíveis.

\section{Referências}

BLACK, N., 1994. Why we need qualitative research. Journal of Epidemiology and Community Health, 48:425-426.

CAMUS, L. B.; ALARCON, E. P.; ORTIZ, F. L.; COLLADO, J. A. M.; CUBERO, P. A. \& BARBAL, M. J. R. A., 1992. Evaluación de la calidad de la asistencia prenatal en atención primaria. Atención Primaria, 10:726-729.

COSTA, J. S. D.; VICTORA, C. G.; BARROS, F. C.; HALPERN, R.; HORTA, B. L. \& MANZOLLI, P., 1996. Assistência médica materno-infantil em duas coortes de base populacional no Sul do Brasil: Tendências e diferenciais. Cadernos de Saúde Pública, 12(Sup. 1):59-56.

DAY, N. E., 1986. The epidemiological basis for evaluation different screening policies. In: Screening for Cancer of the Uterine Cérvix (M. Hakama, A. B. Miller \& N. E. Day, eds.), Scientific Publications 76, pp. 199-212, Lyon: International Agency for Research on Cancer. 
DEAN, J., 1994. EPIINFO - Computer Programs for Epidemiology. Version 6.01. Atlanta: Division of Surveillance and Epidemiology Studies, Epidemiology Programs Office, Centers for Disease Control and Prevention.

DONABEDIAN, A., 1988. The quality of care. How can it be assessed? JAMA, 260:1743-1748.

FORSBERG, B. C.; BARROS, F. C. \& VICTORA, C. G., 1991. Developing countries need more quality assurance: How health facility surveys can contribute. Health Policy and Planning, 7:193-196.

GOLDBAUM, M., 1996. Epidemiologia e serviços de saúde. Cadernos de Saúde Pública, 12(Sup. 2):9598.

GOMES, U. A.; BETTIOL, H.; SILVA, A. A. M.; ALMEIDA, L. E. A.; BARROS, A. A. \& BARBIERI, M. A., 1990. Saúde perinatal em Ribeirão Preto, SP, Brasil: Apresentação de algumas características demógrafo-sociais e da atenção médica da população estudada. Cadernos de Saúde Pública, 6:5-17.

HALPERN, R.; BARROS, F. C.; VICTORA, C. G. \& TOMASI, E., 1998. Atenção pré-natal em Pelotas, Rio Grande do Sul, Brasil, 1993. Cadernos de Saúde Pública, 14:487-492.

HANSELL, M. J., 1991. Sociodemographic factors and the quality of prenatal care. American Journal of Public Health, 81:1023-1028.

KOGAN, M. D.; ALEXANDER, G. R.; KOTELCHUCK, M.; NAGEY, D. A. \& JACK, B. W., 1994. Comparing mothers' reports on the content of prenatal care received with recommend national guidelines for care. Public Health Reports, 109:637-646.

MS (Ministério da Saúde), 1985. Instrumento de avaliação para centros e postos de saúde. Brasília: Centro de Documentação, Ministério da Saúde.

OJEDA, N. S., 1992. Evaluation of maternal and child health services in Latin America. World Health Forum, 13:139-142.
PEOPLES-SHEPS, M. D.; KALSBEEK, W. D.; SIEGEL, E.; DEWEES, C.; ROGERS, M. \& SCHWARTZ, R., 1991. Prenatal records: A national survey of content. American Journal of Obstetrics and Gynecology, 164:514-521.

PETITTI, D. B.; HIATT, R. A.; CHIN, V. \& CROUGHANMINIHANE, C., 1991. An outcome evaluation of the content and quality of prenatal care. Birth, 18:21-25.

SCOCHI, M. J., 1994. Indicadores da qualidade dos registros e da assistência ambulatorial em Maringá (Estado do Paraná, Brasil), 1991: Um exercício de avaliação. Cadernos de Saúde Pública, 10: 356-367.

SSMA (Secretaria da Saúde e do Meio Ambiente do Estado do Rio Grande do Sul), 1997. Programa de Assistência Pré-natal: Detecção e Controle da Gravidez de Alto Risco. Porto Alegre: SSMA.

SMSBE (Secretaria Municipal de Saúde e Bem-estar de Pelotas), 1996. Plano Municipal de Saúde de Pelotas, Rio Grande do Sul. Pelotas: Editora Universitária/Universidade Federal de Pelotas.

SIKOSANA, P. L. N., 1994. An evaluation of the quantity of antenatal care at rural health centres in Metebeleland North Province. Central African Journal of Medicine, 40:268-272.

TAKEDA, S. M. P., 1993. Avaliação de Unidade de Atenção Primária: Modificação dos Indicadores de Saúde e Qualidade da Atenção. Dissertação de Mestrado, Pelotas: Universidade Federal de Pelotas.

TANAKA, A. C. d'A.; SIQUEIRA, A. A. F. \& BAFILE, P. N., 1989. Situação de saúde materna e perinatal no Estado de São Paulo, Brasil. Revista de Saúde Pública, 23:67-75.

UNICEF (United Nations Children's Fund), 1991. A UNICEF Guide for Monitoring and Evaluation: Making a Difference? New York: UNICEF. 